\title{
Contribución al conocimiento de la flora vascular de la desembocadura del río Guadalhorce y su entorno (Málaga, España).
}

\author{
Federico Casimiro-Soriguer Solanas ${ }^{1}$ \& José García-Sánchez ${ }^{2}$ \\ ${ }^{1}$ Dpto. de Biología Vegetal. Facultad de Ciencia. Universidad de Málaga. 29071. Málaga. \\ ${ }^{2}$ Herbario MGC. Servicios Centrales de Apoyo a la Investigación (SCAI). Universidad de Málaga. 29071. Málaga
}

\section{Correspondencia}

F. Casimiro-Soriguer Solanas e-mail: fedeque@hotmail.com

Recibido: 2 noviembre 2017

Aceptado: 10 diciembre 2017

Publicado on-line: diciembre 2017

\begin{abstract}
Resumen
El objetivo de este trabajo es realizar un catálogo florístico de la Desembocadura del Río Guadalhorce y su entorno, que engloba buena parte de la Vega de Málaga y una pequeña franja litoral desde la Desembocadura del Río Guadalhorce hasta el límite con el término municipal de Torremolinos. Toda la zona pertenece al término municipal de Málaga, la cual está fuertemente antropizada, por lo que solo se ha estudiado la flora de las zonas que aún conservan comunidades vegetales naturales. Estas comunidades son todas de carácter edafófilo o higrófilo. Se han recolectado ex profeso para este trabajo 403 pliegos de herbario depositados en el Herbario de la Universidad de Málaga (Herbario MGC). El catálogo final basado en pliegos de herbario se compone de 272 taxones, 21 de los cuales son especies alóctonas agrupadas en un catálogo aparte. Además, se incluye un breve catálogo de citas históricas con 14 taxones que no han podido comprobarse con pliegos de herbario.
\end{abstract}

Palabras clave: Flora vascular, psammofilas, halófilas, higrófilas, Desembocadura Río Guadalhorce, Málaga, Península Ibérica.

\begin{abstract}
Contribution to the knowledge of the vascular flora of the Guadalhorce river mouth and its environment (Malaga, Spain).

The aim of this work is making a floristic catalogue of the Guadalhorce river mouth and its surroundings, which include a high extension of the Malaga floodplain and a small coastal strip from the mouth of the Guadalhorce river to the limit with the municipality of Torremolinos. The whole area belongs to the municipality of Malaga, which is strongly anthropized, so only the flora of the areas that still conserve natural plant communities has been studied. All these communities are edaphophilous or hygrophilous nature. Four hundred and three herbarium sheets included in the University of Malaga Herbarium (MGC Herbarium) have been collected expressly for this work. The final catalogue based on herbarium sheets is composed of 272 taxa, 21 of which are non-native species grouped in an apart catalogue. Moreover, includes a brief catalogue of historical citations with 14 taxa that could not be verified with herbarium sheets.
\end{abstract}

Key words: Vascular flora, psammophilous, halophiles, hygrophilous, Guadalhorce river mouth, Malaga, Iberian Peninsula

\section{Introduccion y objetivos}

El río Guadalhorce desemboca en el Mar de Alborán, en el extremo occidental de la ciudad de
Málaga. En su desembocadura original formaba un delta que se encuentra actualmente totalmente transformado. Esta zona deltaica estuvo compuesta por el río, marjales y zonas arenosas 
conservadas de indudable valor medio ambiental. A partir de la segunda mitad del siglo XIX es cuando empieza una fuerte transformación antrópica: originalmente se produjo una fuerte alteración del medio llevado a cabo en el ámbito de la agricultura con el desarrollo de grandes plantaciones de caña de azúcar, posteriormente con la expansión urbanística de Málaga capital y por último con el encauzamiento de toda la parte final del río. Todas estas actuaciones en dicho enclave de menos de 2.000 hectáreas se han traducido en los últimos años en el desarrollo de grandes áreas urbanas como el núcleo urbano de Churriana, numerosas pedanías, así como urbanizaciones dispersas, un aeropuerto internacional, tres vías de alta capacidad de vehículos, una vía de ferrocarril, seis polígonos industriales, una gran área comercial y un campo de golf (fig. 1).

La fuerte presión humana que soporta el litoral mediterráneo peninsular tiene en el sur de la Península Ibérica un claro exponente y, dentro de los hábitats litorales, seguramente sean los humedales costeros los hábitats más amenazados y escasos. Exceptuando el propio río Guadalhorce y el Paraje Natural Desembocadura del río
Guadalhorce, el resto de localidades estudiadas están llamadas a desaparecer a corto y medio plazo.

Este trabajo se centra en la elaboración de un elenco florístico del área de estudio y no en una flora completa. Se ha decidido así, al ser una zona muy transformada y donde las comunidades naturales se hallan muy localizadas respecto a otras comunidades ruderales muy frecuentes y asociadas a escombreras, bordes de carreteras, plantaciones en zonas más o menos ajardinadas, etc. Se pretende pues recuperar y actualizar el conocimiento de la flora de este enclave.

\section{Antecedentes históricos botánicos}

Las primeras referencias botánicas de la zona con mayor entidad pertenecen a Boissier (1837), que herborizó y comentó el aspecto de la Dehesilla de Málaga, de la que dice: "Hay muy cerca de la ciudad, en la orilla del mar una gran llanura sin cultivar que se extiende una lengua de largo hasta la Desembocadura del Guadalhorce". A continuación, refiere un listado de plantas donde destaca la abundancia de Ononis ramosissima

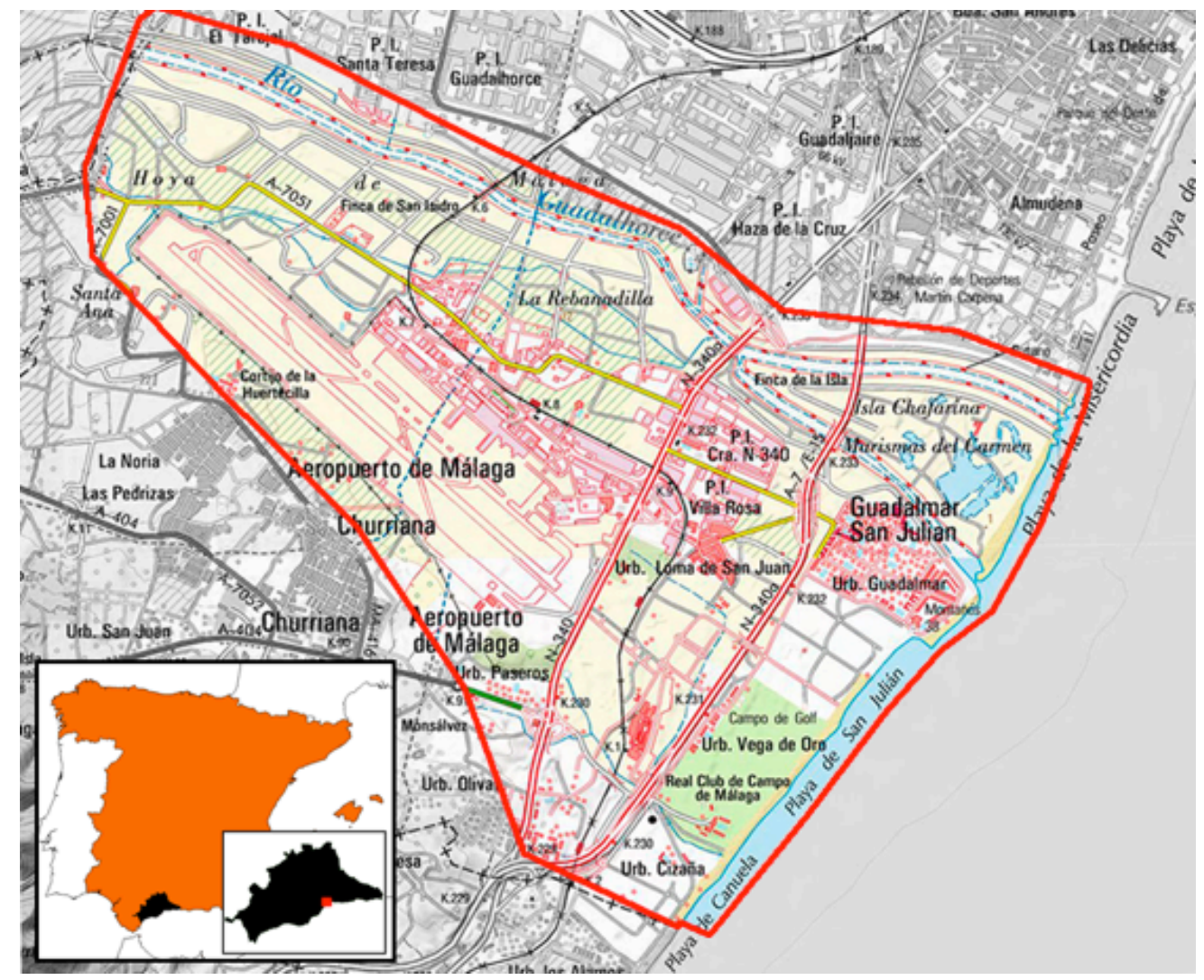

Figura 1. Localización del área de estudio. Modificado a partir del Mapa Topográfico Nacional (MTN50) del Instituto Geográfico Nacional, España, obtenido a través del Visor Iberpix 4, http://www.ign.es/iberpix2/. Figure 1. Location of the study area. Modified from the National Topographic Map (MTN50) of the National Geographical Institute, Spain, obtained through the Iberpix 4 Viewer, http://www.ign.es/iberpix2/ 
Desf. y Festuca alopecurus Schousboe (=Vulpia alopecurus (Schousboe) Dumort.). Posteriormente Willkomm menciona a la Dehesilla como uno de los enclaves en torno a la capital más importantes (Devesa \& Viera, 2001), a la que describe como: "la llanura arenosa y desierta que queda entre la ciudad y el Guadalhorce".

Se convierte así a esta zona en una localidad botánica clásica. Otros botánicos, como Salzmann, Reverchon o Gros también herborizaron en la zona, así como los botánicos locales Haenseler, Prolongo y Laza.

Los únicos trabajos que conocemos en los que aparece nuestra área de estudio, están centrados en la vegetación (Díez Garretas, 1977; Asensi \& Nieto Caldera, 1981). De la Laguna de Los Prados, al estar en el Inventario de Humedales de Andalucía (IHA, Decreto 98/2004, de 9 de marzo de la Junta de Andalucía), existe un pequeño informe sobre sus características botánicas. Está incluida en el IHA por su valor ornitológico basado en la colonia de ardeidos que allí crían, esto último hace problemático acceder a la laguna para herborizar por lo que se optó, de momento, por no incluir este enclave en el estudio.

\section{Material y métodos}

\section{Área de estudio}

La Desembocadura del río Guadalhorce, según Pérez Latorre \& Cabezudo (2002) pertenece a la provincia Bética, sector Malacitano-Axarquiense, subsector Malacitano y de acuerdo a Rivas Martínez et al. (2017), provincia Bética, sector Rondeño, distrito Hoya de Málaga, ambos bajo el ombrotipo seco en el piso termomediterráneo.

Según el Mapa Geológico de España del Instituto Geológico y Minero de España, IGME (Hoja 1744, escala 1:50.000), la mayor parte del área de estudio se asienta en materiales aluviales, exceptuando la playa y los arenales posteriores de naturaleza dunar, siendo todos de origen cuaternario.

El área de estudio abarca buena parte de la Vega de Málaga, conformando una zona muy Ilana, que va desde el nivel del mar hasta los $15 \mathrm{~m}$ s.n.m. en la zona más alejada a este.

Las comunidades vegetales que aún mantienen un cierto grado de conservación son todas de carácter edafófilo o higrófilo, agrupándose en comunidades psammófilas (arenales), halófilas

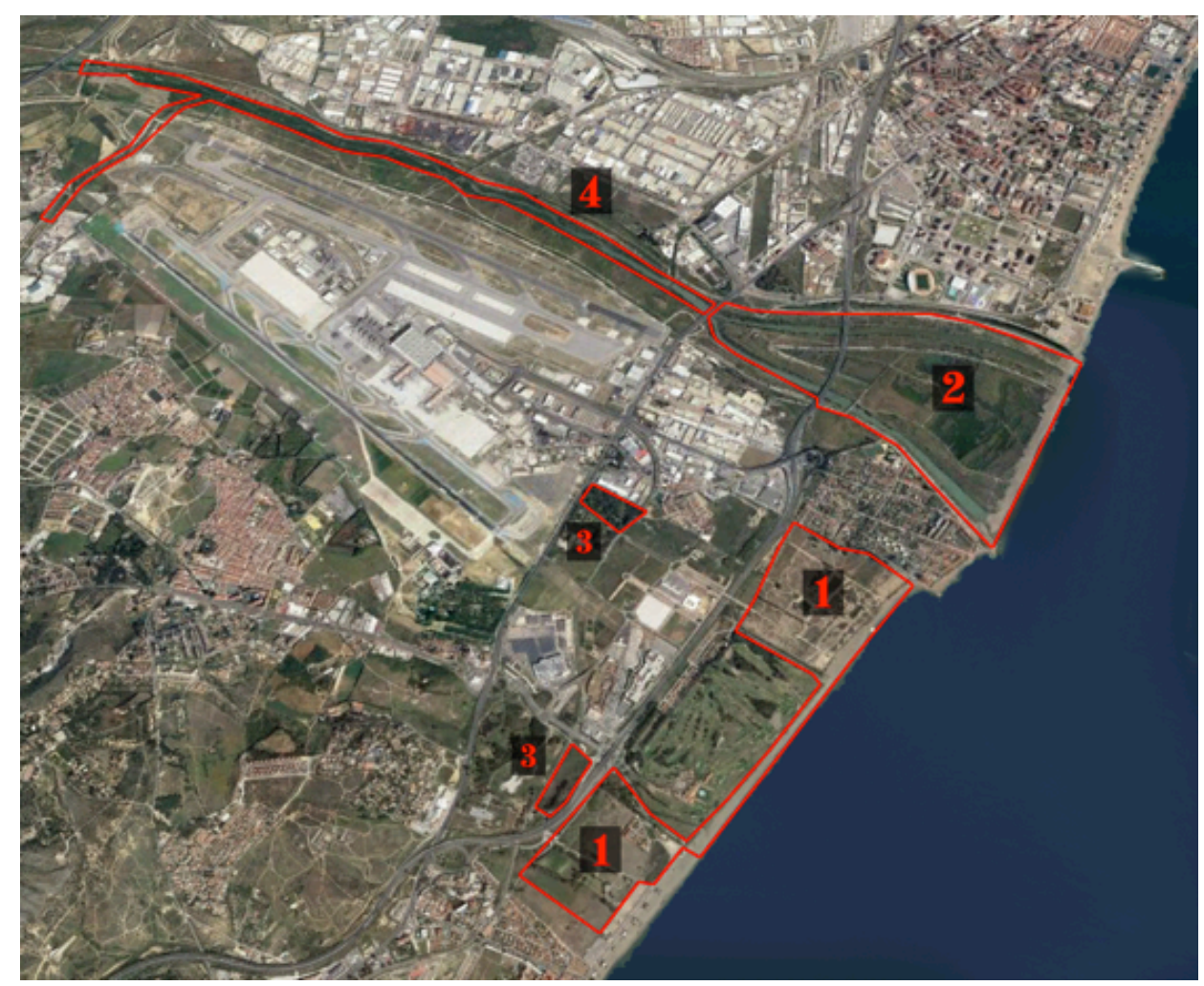

Figura 2. Localización de los enclaves muestreados. 1. Arraijanal-La Cizaña. 2. Paraje Natural Desembocadura del río Guadalhorce. 3. Lagunajos interiores. 4. Río Guadalhorce. Modificado a partir de la Ortofoto (PNOA) del Instituto Geográfico Nacional, España, obtenido a través del Visor Iberpix 4, http://www.ign.es/iberpix2/. Figure 2. Location of the sampled sites. 1. Arraijanal-La Cizaña. 2. Paraje Natural Desembocadura del río Guadalhorce. 3. Lagunajos interiores. 4. Río Guadalhorce. Modified from the Orthophoto (PNOA) of the National Geographic Institute, Spain, obtained through the Iberpix 4 Viewer, http://www.ign.es/iberpix2/ 
(suelos salinos) o higrófilas (comunidades acuáticas de diversa índole, yendo desde comunidades de hidrófitos sumergidos a comunidades arbustivas riparias). Actualmente los enclaves donde aún se pueden localizar estas comunidades vegetales naturales o seminaturales son (fig. 2):

Arraijanal-La Cizaña. Se trata de arenales fijos que comienzan junto a la línea de playa, separada de esta en el mejor de los casos por un pequeño cordón de dunas embrionarias. Esta zona abarca también la Playa del Campo de Golf (parte de la Playa de San Julián), donde estos restos de dunas embrionarias están mejor representadas, aunque su contacto inmediatamente posterior son los céspedes del Parador de Golf. Dentro de estos arenales fijos aparecen juncales y masiegales (formaciones dominadas por la masiega -Cladium mariscus (L.) Pohl-) donde el nivel freático está más próximo a la superficie.

Paraje Natural Desembocadura del río Guadalhorce. La desembocadura del río declarada Paraje Natural por su interés ornitológico, fue posteriormente afectada por el encauzamiento de toda la parte final del río. Al estar protegida toda la zona, el encauzamiento se proyectó de forma que no la destruyese totalmente.

Lo que nos encontramos ahora son pequeñas lagunas procedentes de antiguas excavaciones para la extracción de arenas, grandes superficies de antiguas escombreras, eriales antes ocupados por cultivos de caña de azúcar, el río propiamente dicho dividido en 2 ramales y una antigua zona de marismas junto a la playa.

Lagunajos interiores. Laguna del Campamento Benítez y El lagunajo del Vivero Forestal de San Julián. Se trata de 2 pequeñas lagunas de escasa profundidad que aún se conservan y que formarían junto con la Laguna de los Prados (no incluida en este trabajo), los restos de las zonas húmedas asociadas al antiguo delta del río.

Río Guadalhorce. Comprende el cauce del río desde el límite norte del Paraje Natural hasta el vado del Tarajal, así como el arroyo Ramírez Bienquerido. Ambas cuencas están encauzadas, estando la vegetación riparia en el caso del río recuperada de forma feraz, con comunidades riparias muy densas, pero totalmente desdibujadas. En el caso del arroyo, son las comunidades de grandes heliófilos las que ocupan todo su cauce.

Aunque en la actualidad desaparecida, hay que destacar la Dehesilla de Málaga. Areal al que hacen mención buena parte de las citas históricas de la zona durante el siglo XIX y primera parte del XX, como ya se comentó anteriormente. Este enclave hoy totalmente transformado, ya que forma parte del núcleo urbano de Málaga capital, habría sido otra zona que abarcaría este estudio. Englobaría el margen oriental del río entre la desembocadura y la ciudad, estando formada por arenales y pequeñas zonas húmedas.

\section{Metodología}

Se ha herborizado en las cuatro localidades descritas en el apartado "área de estudio", centrándonos en las comunidades vegetales naturales conservadas, que están asociadas a los siguientes tipos de hábitats:

Comunidades psammófilas. Vegetación localizada tanto en las playas como en los arenales fijos posteriores.

Comunidades halófilas y halonitrófilas. Vegetación que ocupa las zonas de suelos con cierta acumulación de sal, pudiendo ser suelos de textura limosa, arcillosa o arenosa.

Comunidades dulceacuícolas o hiposalinas. Vegetación asociada a las masas de agua dulce o con una salinidad moderada, como son los lagunajos interiores, arroyos y el mismo río.

Comunidades acuáticas de aguas salinas. Vegetación asociada a las masas de agua muy salinas que en la zona están representadas por la mayoría de lagunajos que forman el Paraje Natural.

El catálogo florístico final es principalmente el resultado de las recolecciones realizadas entre los años 2015 y 2017. Además, se han incluido registros de flora procedentes de búsquedas en el Portal nacional online de la Infraestructura Mundial de Información en Biodiversidad (GBIF) del material recolectado en la zona (http://datos. gbif.es, 24/01/2013), así como citas bibliográficas históricas del área de estudio.

Para la identificación y nomenclatura del material recolectado, se han seguido fundamentalmente las obras "Flora Vascular de Andalucía Oriental" (Blanca et al., 2011), "Flora iberica" (Castroviejo et al., 1986-2015) y "Flora Vascular de Andalucía Occidental" (Valdés et al., 1987). Para las gramíneas ha sido de gran utilidad la monografía de Romero-Zarco (2015). Para la nomenclatura de Thrincia hemos consultado a Talavera et al. (2015), para Hedypnois a Talavera et al. (2016) y en el caso de Arundo a Hardion et al. (2012).

El catálogo florístico resultante se presenta en 3 partes: un breve catálogo, que es una compilación 
de citas históricas para táxones sin respaldo de pliegos ni recolecciones recientes, un catálogo principal basado en pliegos de herbario, teniendo como base la colección de cormófitos del herbario de la Universidad de Málaga (MGC), así como diversas colecciones de herbarios españoles (MA, GDA, GDAC, COA, COFC, JBAG, SALA, VAL, BC, Anthos), todas ellas consultadas a través del Portal de datos de GBIF y un último catálogo donde se seleccionan las especies alóctonas. Mientras no se indique lo contrario, todos los taxones tienen al menos un pliego testigo en el Herbario MGC. En el catálogo principal, los pliegos de referencia en los que se indica como recolector "F. Soriguer", se refiere a uno de los autores de este trabajo: "F. Casimiro-Soriguer Solanas".

Para el catálogo principal, la información que se presenta para cada taxón es: nombre científico; siglas de la colección de los pliegos de herbario de referencia; localidad (indicándose una de las cuatro en las que dividimos el área de estudio); hábitat y abundancia en la zona (según Blanca et al., 2011). Para las citas históricas: nombre científico; referencia bibliográfica y además, para la mayoría una pequeña discusión. Por último, para el catálogo de alóctonas, donde tomamos de referencia a Dana et al. (2005) y Casimiro-Soriguer Solanas \& Pérez Latorre (2008), la información expuesta para cada taxón es: nombre científico; siglas de la colección del pliego o pliegos de herbario de referencia; localidad (una de las cuatro en las que dividimos la zona de estudio); hábitat; abundancia en la zona y tipo de xenófito.

Para los taxones que consideramos que son novedad corológica o muy raros en el territorio, incluimos todos los datos de los pliegos testigos. Estas novedades corológicas se basan principalmente en las comarcas naturales según Flora de Andalucía Oriental y las provinciales de Flora iberica. También añadimos un pequeño comentario en los taxones donde creemos necesario aclarar algún dato corológico y/o taxonómico.

Por último, hemos resaltado algunos taxones incluídos en la Lista Roja de la Flora Vascular de Andalucía (LRA) (Cabezudo et al., 2005) con alguna de las siguientes categorías: EN (en peligro), VU (vulnerable), DD (datos insuficientes), así como a taxones incluídos en el Listado Andaluz de Especies Silvestres en Régimen de Protección (LAESRP) según el Decreto 23/2012 del 14 febrero de la Junta de Andalucía.

\section{Resultados}

Para el desarrollo del catálogo principal, se hicieron un total de 18 recolecciones entre los años 2015 y 2017, reuniéndose un total de 403 pliegos de herbario. Todo este material, junto con el ya existente previamente en los fondos del herbario MGC (253 ejemplares), así como la información del resto de colecciones consultadas en GBIF (101 registros) y las citas históricas, reúnen un total de 286 taxones (222 recolectados por alguno de los autores de este trabajo), pertenecientes a 174 géneros y 54 familias.

Toda la información de este estudio realacionada con su metodología, área geográfica y catálogo florístico, se ha resumido en una publicación tipo checklist que estará disponible online a través del portal de datos de GBIF y que estára disponible en la siguiente dirección web tras la publicación de este trabajo: https://www.gbif.org/ dataset/ab208927-fbf3-4994-b2fe-14cb6c31aed5

\section{Citas históricas}

Recopilación de taxones citados en la zona de estudio y que son característicos de los hábitats prospectados. Son referencias sin pliego de herbario o bien con pliego pero que no hemos podido revisar.

\section{CARYOPHYLLACEAE}

\section{Loeflingia hispanica $\mathrm{L}$.}

Willkomm \& Lange (1861-1880, III: 159), recogen el testimonio de diversos autores sobre la presencia de esta planta en los arenales próximos a Málaga

\section{Polycarpon alsinifolium (Biv.) DC}

Boissier (1839-1842, II: 221), cita esta especie de la zona. Todo el material de este género recolectado por nosotros y que presenta semillas maduras se corresponde con $P$. tetraphyllum, siendo la subespecie más abundante en los arenales de la zona $P$. tetraphyllum subsp. dyphyllum

\section{Silene echinata Otth}

En la Península Ibérica esta Silene solo aparece citada en las proximidades de Málaga, en arenales marítimos. Cita seguramente referida a un pliego de Boissier (Talavera \& Muñoz Garmendia, 1988). Estos mismos autores indican la probabilidad de que sea una planta adventicia. Nosotros no hemos encontrado referencias posteriores ni hemos conseguido localizarla para este trabajo, por lo que pensamos que es una especie a excluir de la flora ibérica.

\section{Silene littorea Brot.}

Las únicas referencias encontradas son de 
Rivas Goday \& Rivas Martínez (1959) y Díez Garretas (1977), que la citan de la Desembocadura del Guadalhorce, aunque no hemos encontrado pliegos testigo. De los autores clásicos del XIX no hemos encontrado referencias. Nosotros no la hemos visto pese a ser una planta realmente vistosa en floración, por lo que no creemos que actualmente siga habitando en la zona

\section{CONVOLVULACEAE}

Calystegia soldanella (L.) $\mathrm{R}$. Br.

La única referencia encontrada es de Rivas Goday \& Rivas Martínez (1959) que la citan en la Desembocadura del Guadalhorce. De los autores clásicos del XIX no hemos encontrado referencias. Tampoco Silvestre (2012) en Flora iberica recoge esta cita (ni como extinta). La cita nos parece dudosa y nosotros no la hemos visto pese a ser una planta muy característica.

\section{GRAMINEAE}

Cutandia divaricata (Desf.) Barbey

Cabezudo et al. (1990) y Romero (2011), citan esta especie para Málaga, basándose en la cita de Boissier (1839-1842, II:666) de Sclerochloa divaricata Pal. Este último autor recoge en la sinonimia de este taxón a Poa divaricata Gouan y Festuca expanda Kunth. Posteriormente Willkomm \& Lange (1861-1880. I: 86), recogen esos tres nombres (S. divaricata, $P$. divaricata y F. expanda), en la sinonimia de Sphenopus gouanii Trin. Estas citas se basan en recolecciones de Haenseler, "cerca de Málaga en arenales marítimos". Parece entonces que Cutandia divaricata (Desf.) Barbey, no fue realmente lo que recolecto Haenseler sino lo que ahora se acepta como Sphenopus divaricatus (Gouan) Rchb. (=Sphenopus gouanii Trin.)

No hemos encontrado referencias posteriores $y$, solo de manera muy localizada, hemos herborizado Cutandia maritima. Pensamos por tanto que Cutandia divaricata es una especie a excluir de la flora ibérica, puesto que es esta la única cita peninsular de la que tenemos constancia (Romero Zarco, 2015).

Hemarthria altissima (Poir.) Stapf et C.E. Hubb.

Recolectada por Haenseler en la Dehesilla y aledaños a Málaga, en arenales marítimos húmedos y juncales (Willkomm \& Lange, 18611880, I: 116; Boissier, 1839-1842, II:684, sub. $H$. fasciculata Kunth). No hemos encontrado referencias posteriores. La referencia en Flora de Andalucía Oriental para Axarquía, creemos que se basa en las citas anteriores. Aunque la hemos buscado sin éxito, si esta planta aun habita en la zona, debe encontrarse en las orillas del Guadalhorce, de difícil acceso debido a la densidad de la vegetación riparia.

Sphenopus divaricatus (Gouan) Rchb.

Willkomm \& Lange (1861-1880, I: 86 sub. Sphenopus gouani Trin) y Boissier (1839-1842, II: 666 sub. Schlerochloa divaricata Pal), basándose en Haenseler, citan la especie en la zona en suelos arenosos húmedos y salinos. Cabezudo et al. (1990), recogen para la zona también una cita de Laza (1944). No hemos encontrado referencias posteriores.

Aeluropus littoralis (Gouan) Parl

Willkomm \& Lange (1861-1880, I: 87) y Boissier (1839-1842, II: 664 sub. Dactylis littoralis Willd.) basandose en Haenseler, recogen la presencia de esta especie en las cercanias de Málaga, en arenales marítimos.

\section{FRANKENIACEAE}

\section{Frankenia corymbosa Desf.}

Cita recogida por Willkomm (1893: 286), como F. webbii Boiss. \& Reut., en Málaga, en arenales marítimos, basándose en un pliego de Reverchon. En la zona de estudio la única especie perenne del género que hemos encontrado es $F$. laevis.

\section{LEGUMINOSEAE}

\section{Lotus creticus $\mathrm{L}$.}

Boissier (1839-1842, II: 175), cita esta planta en las playas de Málaga. Nosotros en esta ecología solo hemos herborizado $L$. arenarius

\section{JUNCACEAE}

Juncus striatus Schousb. ex E. Mey.

Junco perenne, frecuente en Andalucía (Romero Zarco, 2010), pero del que solo tenemos constancia de una cita anterior en la zona (Willkomm \& Lange, 1861-1880, I: 184)

\section{UMBELIFERAE}

\section{Berula erecta (Huds.) Coville}

Tanto Willkomm \& Lange (1861-1880, III: 95) como Boissier (1939-1842, II: 244), ambos como Sium angustifolium L., y fundamentándose en Haenseler, la citan en zonas húmedas cercanas a la capital. Aunque Flora iberica y Flora de Andalucía Oriental citan la especie para Málaga, al parecer no han podido consultar ningún pliego testigo. 


\section{Cahrys libanotis $\mathrm{L}$.}

Cabezudo et al. (1992), recogen el testimonio de Prolongo. No hemos encontrado más referencias, y todo lo visto por nosotros en pliegos y en el campo corresponde a $C$. sicula $\mathrm{L}$.

\section{Catálogo principal}

En el catálogo que se presenta a continuación, las abreviaturas que se utilizan son las siguientes:

Localidades: Arraijanal-La Cizaña (Arraij.); Paraje Natural Desembocadura del río Guadalhorce (PN_Desemb.); Lagunajos interiores (Lags.); Río Guadalhorce (RioG.)

Abundancia. Muy rara (rr.); rara (ra.); ocasional (oc.); frecuente (fr.); común (co.)

\section{PTERIDOFITOS EQUISETACEAE}

\section{Equisetum ramosissimum Desf.}

Arraij. PN_Desemb. Lags. RioG.; Pastizales sobre suelos arenosos húmedos; $r$ r.

\section{ANGIOSPERMAS AIZOACEAE}

\section{Mesembryanthemum nodiflorum $\mathrm{L}$.} oc.

Arraij. PN_Desemb.; Pastizales halonitrófilos;

\section{ALISMATACEAE}

\section{Alisma lanceolatum With.}

Lags.; Formaciones de heliofilos en aguas dulces; rr.

Aunque recolectada en diversos puntos de la zona, nosotros solo la hemos visto y herborizado en la Laguna del Vivero de San Julián.

\section{AMARYLLIDACEAE}

\section{Pancratium maritimum $L$.}

Arraij., PN_Desemb; Pastizales sobre arenas fijas; oc.; NT(LRA)

Sorprendentemente sin pliego testigo. Citada por Diez Garretas (1977) y vista por nosotros en todos los arenales de la zona.

\section{APOCYNACEAE}

\section{Vinca difformis Pourret}

PN_Desemb., Lags., Rio G.; Vegetación riparia; oc.

\section{ASCLEPIADACEAE}

\section{Cynanchum acutum $\mathrm{L}$.}

Málaga. Málaga. Paraje Natural Desembocadura del Guadalhorce. Arenales consolidados entre las lagunas y la playa. 30SUF7059. 27/4/2015. F. Soriguer \& J. M. Nieto. MGC 80730

PN_Desemb.; Vegetación sobre arenas fijas; rr.

Planta muy rara en Málaga. Por lo que sabemos, esta es su única localidad en la provincia, no estando citada para la Axarquía en Flora de Andalucía Oriental.

\section{BORAGINACEAE}

\section{Anchusa calcarea Boiss. subsp. calcarea}

Málaga. Málaga. La Cizaña. 30SUF6757. 2/4/2009. B. Cabezudo, A. V. Pérez Latorre \& F. Soriguer. MGC 69428; Torremolinos. La Cizaña. Entre la Casa de la Cizaña y la Urbanización La Cizaña. 30SUF6756. 30/03/2016. Arenales litorales consolidados. Pastizales. F. Soriguer. MGC 81926; Torremolinos. La Cizaña. Entre la Casa de la Cizaña y la Urbanización Los Álamos. 30SUF6756. 30/03/2016. Arenales litorales consolidados. Pastizales. F. Soriguer. MGC 81914

Arraij.; Pastizales sobre arenas fijas; oc.; DD(LRA)

Especie propia de arenales costeros que en Andalucía oriental solo está presente en la provincia de Málaga (Valdés, 2012), estando recogida en Flora de Andalucía Oriental solo para el Aljibe. Las poblaciones de la zona son por lo tanto las más surorientales de la especie.

Heliotropium europaeum $\mathrm{L}$.

PN_Desemb.; Pastizales ruderalizados sobre arenas; oc.

\section{CAMPANULACEAE}

Jasione blepharodon Boiss. et Reut.

Arraij.; Pastizales sobre arenas fijas; ra.

\section{CAPRIFOLIACEAE}

Lonicera biflora Desf. (LRA)

PN_Desemb.; Vegetación riparia; ra.; DD

Incluimos con reservas en el catálogo principal a esta madreselva porque su carácter autóctono en la zona es dudoso (Casimiro-Soriguer Solanas, 2009) 


\section{CARYOPHYLLACEAE}

Herniaria cinerea DC.

Arraij.; Pastizales sobre arenas fijas y pastizales halonitrófilos; ra.

Paronychia argentea Lam.

Arraij.; Pastizales sobre arenas fijas; fr.

Polycarpon tetraphyllum subsp. diphyllum (Cav.) O. Bolòs et Font Quer

Arraij. PN_Desemb.; Pastizales sobre arenas; fr.

Polycarpon tetraphyllum $\quad$ (L.) L. subsp.
tetraphyllum
Arraij. PN_Desemb.; Pastizales sobre arenas; oc.

Silene gallica $\mathrm{L}$.

PN_Desemb.; Pastizales sobre arenas fijas; ra.

Silene micropetala Lag.

Arraij.; Pastizales sobre arenas fijas; oc.

Silene nicaeensis All.

Arraij. PN_Desemb.; Pastizales sobre arenas; co.

\section{Silene sclerocarpa Dufour}

Arraij.; Pastizales sobre arenas fijas; co.

Spergularia bocconei (Scheele) Ash. et Graebn. PN_Desemb.; Pastizales halonitrófilos; ra.

\section{Spergularia heldreichii Foucaud}

Arraij. PN_Desemb.; Pastizales halonitrófilos; ra.

Spergularia marina (L.) Besser

Arraij. PN_Desemb.; Pastizales halonitrófilos; oc.

Spergularia nicaeensis Sarato ex Burnat Arraij.; Pastizales halonitrófilos; oc.

\section{CHENOPODIACEAE}

Atriplex patula $\mathrm{L}$.

PN_Desemb.; Pastizales halonitrófilos; oc.

Atriplex prostrata Boucher ex DC.

PN_Desemb.; Pastizales halonitrófilos; oc.

Bassia hyssopifolia (Pall.) Kuntze

PN_Desemb.; Pastizales halófilos; ra.
Beta macrocarpa Guss.

Arraij.; Pastizales halófilos; ra.

Beta maritima L.

Arraij., PN_Desemb.; Pastizales halófilos; ra.

Chenopodium ambrosioides $\mathrm{L}$.

PN_Desemb.; Pastizales ruderalizados sobre arenas; ra.

\section{Chenopodium glaucum $\mathrm{L}$.}

PN_Desemb., Arrij.; Pastizales halófilos sobre suelos encharcados; oc.

Chenopodium murale $\mathrm{L}$.

Arraij. PN_Desemb.; Pastizales ruderalizados sobre arenas; ra.

Halimione portulacoides (L.) Aellen

PN_Desemb.; Pastizales halófilos; co.

Por lo que sabemos, estas poblaciones son las únicas de la provincia de Málaga

Patellifolia patellaris (Moq.) A.J. Scott, Ford-Lloyd et J.T. Williams

Arraij. PN_Desemb.; Pastizales ruderalizados sobre arenas; oc.

\section{Salicornia ramosissima Woods}

Málaga. Málaga. El Arraijanal. Arroyo en el límite oriental del campo de golf. 30SUF6857. 10/10/2017. Comunidades de anuales en suelos húmedos sometidos a la influencia marina. $F$. Soriguer, J. García-Sánchez \& M. Pulgar. MGC 87348; Ibidem. 30SUF6858. 21/07/2016. Arroyo. Vegetación higrófila. F. Soriguer. MGC 84068

Arraii.; Pastizales halófilos; rr.

No tenemos referencias anteriores de su presencia en la zona (Díez Garretas, 1977; Asensi \& Nieto, 1981). No citada en Flora de Andalucía Oriental para la Axarquía

\section{Salsola kali L.}

Arraij. PN_Desemb.; Pastizales ruderalizados sobre arenas; $\mathrm{fr}$.

Sarcocornia fruticosa (L.) A. J. Scott.

Málaga. Marais maritimes. 1888. E. Reverchon. MA 29447

Málaga. Málaga. Paraje Natural Desembocadura del Guadalhorce. Antiguas marismas del Carmen. 30SUF6958. 15/09/2016. Pastizales halo-nitrófilos. F. Soriguer. MGC 84107

PN_Desemb.; Pastizales halófilos; rr.

De los dos almajos que crecen en la zona, este es el más raro, estando localizado en las antiguas 
marismas del Carmen. Nosotros solo hemos localizado un ejemplar claramente identificable tanto por el porte como por la ornamentación de las semillas. Este crece entre las densas formaciones del otro almajo de la zona ( $S$. perennis subsp. alpini). Debió ser más común antes de las fuertes transformaciones de la zona, aunque solo hemos encontrado una cita anterior (Castroviejo \& Coello, 1980) basada en la recolección de Reverchon, aunque después Castroviejo (1990) no recoge su presencia en Málaga. En la zona estaba citada como Arthrocnemum fruticosum (L.) Moq., por Diez Garretas (1977) y Asensi \& Nieto (1981), aunque en realidad los pliegos recolectados por los autores corresponden a la especie siguiente.

No citada en Flora de Andalucía Oriental para Axarquía, ni para ninguna otra unidad que abarque a la provincia de Málaga

$\begin{gathered}\text { Sarcocornia } \\ \text { Castrov. } \\ \text { Málaga. }\end{gathered}$ Málaga. Desemnis subsp. alpini (Lag.) Guadalhorce. 30SUF7016059385. 15/2/1994. D. Navas \& A. Pérez-Latorre. MGC 37416; Ibidem. 30SUF70095918. 19/3/2002. J. M. Nieto, J. L. Díaz, R. Conde \& O. Comino. MGC 52410; Málaga; Paraje Natural Desembocadura del Guadalhorce. En la zona de las antiguas Marismas del Carmen. Entre el brazo derecho del río y las lagunas. 30SUF7059. 27/4/2015. F. Soriguer \& J. M. Nieto. MGC 80719; Ibidem. Antiguas marismas del Carmen. 30SUF6958. 15/09/2016. Pastizales halo-nitrófilos. F. Soriguer. MGC 84109; Ibidem. Entre la laguna grande y el cauce occidental del río. 30SUF6959. 15/09/2016. Pastizales halo-nitrófilos. F. Soriguer. MGC 84102; Ibidem. Laguna de la caseta. Borde del agua. 30SUF6959. 15/09/2016. Pastizales halo-nitrófilos. F. Soriguer. MGC 84116; Ibidem. Laguna Grande. Bordel del agua. 30SUF6959. 15/09/2016. Pastizales halonitrófilos. F. Soriguer. MGC 84115

PN_Desemb.; Pastizales halófilos; co.

El almajo más abundante de la zona, que se ha visto favorecido por la salinización de las lagunas del Paraje Natural. Recientemente reivindicada como especie independiente, como S. alpini (Lag.) Rivas Mart., en la práctica es muy difícil separarla de S. perennis (Miller) A.J. Scott, por lo que optamos por seguir a Castroviejo (1990) en Flora iberica. En Flora de Andalucía Oriental (Cabello, 2011) solo la cita para Almería (Punta Entinas), aunque comenta que estaba citada para Málaga, pero no pudo consultar pliegos de herbario.

Suaeda spicata (Willd.) Moq.

Málaga. Málaga. Paraje Natural
Desembocadura del Guadalhorce. En la zona de las antiguas Marismas del Carmen. Entre el brazo derecho del río y las lagunas. 30SUF7059. 27/4/2015. Vegetación halófila ruderalizada. F. Soriguer \& J. M. Nieto. MGC 80720; Ibidem. Marismas del Carmen. Antigua zona marismeña. 30SUF7058. 19/5/2015. Vegetación halófila ruderalizada. F. Soriguer. MGC 80880; Ibidem. Entre la laguna grande y el cauce occidental del río. 30SUF6959. 15/09/2016. Pastizales halonitrófilos. F. Soriguer. MGC 84104; Ibidem. Antiguas marismas del Carmen. 30SUF6958. 15/09/2016. Pastizales halo-nitrófilos. F. Soriguer. MGC 84112. MGC 84111

PN_Desemb.; Pastizales halófilos y halonitrófilos; oc.

No recogida para la Axarquía ni para ninguna unidad incluida en la provincia de Málaga en Flora de Andalucía Oriental, aunque Castroviejo (1990), si la cita para Málaga. De la zona estudiada ya estaba citada por Diez Garretas (1977) como S. maritima (L.) Dumort., siendo esta la única localidad de la especie en la provincia.

\section{CISTACEAE}

Helianthemum salicifolium (L.) Mill. Arraij.; Pastizales sobre arenas fijas; oc.

\section{COMPOSITAE}

Aetheorhiza bulbosa (L.) Cass. subsp. bulbosa Arraij.; Pastizales sobre arenas; oc.

Anacyclus radiatus Loisel. subsp. radiatus Arraij.; Pastizales sobre arenas; co.

Andryala integrifolia $\mathrm{L}$.

Arraij.; Pastizales sobre arenas; ra.

Asteriscus maritimus (L.) Less.

Arraij.; Pastizales sobre arenas fijas; rr.

Calendula aegyptiaca Desf.

Arraij.; Pastizales sobre arenas fijas; ra.

Centaurea sphaerocephala L. oc.

Arraij.; pastizales ruderalizados sobre arenas;

Glebionis coronaria (L.) Cass. ex Spach

PN_Desemb.; Pastizales halonitrófilos; oc.

Hedypnois arenaria (Schousb.) DC.

Málaga. Málaga. La Cizaña. 30SUF6756. 04/05/2016. Arenales litorales semimóviles 
ruderalizadas. F. Soriguer. MGC 82658; Ibidem. Entre la Urbanización La Cizaña y la playa. 30SUF6856. 04/05/2016. Arenas litorales. Pastizales sobre arenas. F. Soriguer. MGC 82671; Torremolinos. La Cizaña. Entre la casa de La Cizaña y la Urbanización de los Álamos. 30SUF6756. 06/04/2016. Arenales litorales. Pastizales sobre arenas consolidadas. J. García-Sánchez \& F. Soriguer. MGC 82106; Málaga. Margen oriental del Campo de Golf. 30SUF6857. 28/04/2016. Arenales estabilizados. F. Soriguer. MGC 82500

Arraij.; Pastizales sobre arenas fijas; oc.

Planta propia de arenales costeros, muy rara en Andalucía oriental según Blanca (2011) y circunscrita según el mismo autor al Aljibe.

Hedypnois cretica (L.) Dum. Cours.

Málaga. Málaga. La Cizaña. Entre la Urbanización La Cizaña y la playa. 30SUF6856. 04/05/2016. Arenas litorales. Pastizales sobre arenas. F. Soriguer. MGC 82663; Málaga. Playa del Campo de Golf. 30SUF6857. 14/04/2016. Dunas embrionarias. Arenas. F. Soriguer. MGC 82267

Arraij.; Pastizales sobre arenas; oc.

Incluimos este taxón también propio de pastizales costeros siguiendo a Talavera et al. (2016).

Hedypnois rhagadioloides (L.) F. W. Schmidt Arraij.; Pastizales sobre arenas; oc.

Helminthotheca comosa (Boiss.) J. Holub subsp. comosa

Arraij.; Pastizales graminoides sobre suelos arenosos húmedos; ra.

Helminthotheca echioides (L.) Lack

PN_Desemb.; Pastizales halonitrófilos; ra.

Hypochaeris glabra L.

Arraij. PN_Desemb.; Pastizales sobre arenas fijas; fr.

Otanthus maritimus (L.) Hoffmanns. et Link Arraij. PN_Desemb. Lags.; Pastizales sobre arenas moviles (playas); fr.

Phagnalon rupestre (L.) DC.

Arraij.; Pastizales sobre arenas fijas; ra.

Phagnalon saxatile (L.) Cass.

Arraij.; Pastizales sobre arenas fijas; ra.

Pulicaria paludosa Link

Arraij.; Pastizales sobre suelos arenosos húmedos; oc.
Reichardia tingitana (L.) Roth

Arraij. RioG.; Pastizales sobre arenas; fr.

Sonchus oleraceus $\mathrm{L}$.

PN_Desemb.; pastizales halófilos; oc.

\section{Sonchus tenerrimus $\mathrm{L}$.}

Arraij. PN_Desemb.; Pastizales sobre arenas; oc.

Thrincia hispida (Roth) Roth

Arraij. PN_Desemb.; Pastizales sobre arenas; fr.

Thrincia tingitana Boiss. et Reut.

Arraij.; Pastizales graminoides sobre suelos arenosos húmedos; rr.

\section{Tragopogon porrifolius $\mathrm{L}$.}

Arraij.; Pastizales graminoides sobre suelos arenosos húmedos; ra.

Xanthium strumarium subsp. cavanillesii (Schouw) D. Löwe et P. Dansereau

PN_Desemb.; Pastizales sobre suelos arenosos húmedos; oc.

\section{CONVOLVULACEAE}

Calystegia sepium (L.) R. Br.

Arraij. PN_Desemb.; Vegetación riparia; fr.

\section{CRUCIFERAE}

\section{Brassica tournefortii Gouan}

Arraij.; Pastizales sobre arenas fijas; oc.

\section{Cakile maritima Scop. subsp. maritima}

Arraij. PN_Desemb.; Pastizales sobre arenas moviles (playas); co.

Cardaria draba (L.) Desv.

PN_Desemb.; Vegetación riparia; ra.

Coronopus squamatus (Forssk.) Asch.

PN_Desemb.; Pastizales sobre suelos húmedos; ra.

Hirschfeldia incana (L.) Lagr.-Foss.

Arraij.; Pastizales sobre arenas fijas; fr.

Lobularia maritima (L.) Desv. subsp. maritima Arraij.; Pastizales sobre arenas; co.

Malcolmia littorea (L.) R. Br.

Arraij.; Pastizales sobre arenas fijas; co.

Raphanus raphanistrum $\mathrm{L}$.

Arraij.; ra. 
Rorippa nasturtium-aquaticum (L.) Hayek Lags.; oc.

\section{CYPERACEAE}

Bolboschoenus maritimus Palla

Arraij. PN_Desemb. Lags. RioG.; Comunidades de grandes helófitos (bayuncales); co.

Carex cuprina (I. Sándor ex Heuff.) Nendtv. Arraij. Lags. RioG.; Juncales y herbazales sobre suelos humedos; oc.

\section{Carex divisa Hudson}

Lags.; Juncales y herbazales sobre suelos humedos; ra.

\section{Carex extensa Gooden.}

Málaga. Málaga. La Cizaña. Entre la Urbanización La Cizaña y la playa. 30SUF6856. 20/05/2016. Juncales y masiegales. Suelos arenosos encharcados. F. Soriguer. MGC 82740; 20/05/2016. Juncales y masiegales. Suelos arenosos encharcados. F. Soriguer. MGC 82740

Arraij.; Juncales y herbazales halófilos sobre suelos arenosos humedos; rr.

Cárice raro y localizado en Andalucía (Silvestre, 1987; Salazar \& Quesada, 2011). En Málaga estaba citado por Boissier (1839-1842 II: 631) y Willkomm \& Lange (1861-1880, I: 128), en la Dehesilla de Málaga, en zonas arenosas húmedas, aunque Luceño \& Escudero (2007) en Flora iberica no recogen su presencia en Málaga. Solo hemos encontrado una pequeña población en La Cizaña, ocupando su hábitat típico. Por ahora es la única localidad de la especie en la provincia.

\section{Carex hispida Willd.}

Arraij. Lags.; Pastizales graminoides sobre suelos arenosos húmedos; ra.

\section{Carex riparia Curtis}

Málaga. Málaga. San Julián. Entre el vivero del ICONA y el polígono industrial. Lagunajo del vivero. 30SUF6758. 29/03/2017. Formaciones de helófitos y grandes carices sobre suelos encharcados arcillosos. F. Soriguer \& J. GarcíaSánchez. MGC 84815; San Julián. Los Viveros. Parcela entre el vivero de la Junta de Andalucía y el polígono industrial. 30SUF6758. 17/05/2016. Laguna de aguas permanentes. Comunidad de grandes carices muy desarrolladas. F. Soriguer. MGC 82708

Lags.; Comunidades de grandes cárices asociados a aguas dulces; ra.

Cárice muy raro y localizado en Andalucía. En
Andalucía occidental, Silvestre (1987), solo lo cita de una sola localidad del litoral onubense, Luceño (2007) en Flora iberica solo recoge su presencia en Andalucía en Huelva, mientras que Salazar \& Quesada (2011), recogen una sola localidad para Andalucía oriental, en Sierra Tejeda (MGC 58571). Solo hemos encontrado una población en la Laguna del Vivero de San Julián, donde forma una comunidad de gran talla (hasta 2 m aprox.), prácticamente monoespecífica.

\section{Cladium mariscus (L.) Pohl}

Málaga. Málaga. La Cizaña. Entre la Urbanización La Cizaña y la playa. 30SUF6856. 20/05/2016. Juncales y masiegales. Suelos arenosos encharcados. F. Soriguer. MGC 82744; La Cizaña. Zona con encharcamiento temporal entre la playa y la Urbanización La Cizaña. 30SUF6856. 30/03/2016. Arenales litorales. Juncales y masiegales. F. Soriguer. MGC 81934

Arraij.; Comunidades de grandes helófitos (masiegales) sobre suelos arenosos encharcados; rr.

Cyperácea de gran talla rara en Andalucía sobre todo en su mitad oriental. En Málaga solo conocemos una referencia anterior, en Manilva (MGC 49775), en la que se basa Salazar (2011) para indicar su presencia en el Aljibe en Flora de Andalucía Oriental. Solo hemos encontrado una población en La Cizaña, donde forma una comunidad monoespecífica de gran talla.

\section{Cyperus capitatus Vand.}

Arraij.; Pastizales sobre arenas fijas; ra.

\section{Cyperus distachyos All.}

PN_Desemb.; Comunidades de helófitos en el borde del río; ra.

\section{Cyperus fuscus $\mathrm{L}$.}

Arraij.; juncales y herbazales sobre suelos encharcados; rr.

Eleocharis palustris (L.) Roem. et Schult.

Lags.; Comunidades de helófitos en la desembocadura del río; rr.

\section{Eleocharis uniglumis (Link) Schult.}

Málaga. Málaga. Entre Campo de Golf y Campamento Benítez. 30SUF65. 09/06/1980. J. M. Nieto. MGC 8667

Lags.; Comunidades de helófitos en la desembocadura del río; rr.

Cyperacea muy rara en Andalucía Oriental (Salazar, 2011), cuyas citas para territorio malagueño corresponden a esta localidad. En 
nuestras prospecciones no hemos vuelto a localizar ningún Eleocharis spp. en la zona.

Schoenoplectus lacustris subsp. glaucus (Sm.) Luceño et Marín

Arraij., RioG.; Comunidades de grandes helófitos (bayuncales y espadañales); oc.

Schoenoplectus litoralis (Schrad.) Palla

PN_Desemb.; Comunidades de helófitos en la desembocadura del río; rr.

\section{Scirpoides holoschoenus (L.) Soják} Arraij., RioG, PN_Desemb.; juncales; co.

\section{DIPSACACEAE}

Pycnocomon intermedium (Lag.) Greuter et Burdet

Arraij.; Pastizales sobre arenas fijas; ra.

Parece que esta población es la más oriental de este endemismo ibérico, citado recientemente en la zona (Casimiro-Soriguer Solanas et al., 2013), aunque probablemente fuera herborizado antes por Haenseler, que indica: "Málaga, ...in arenosis regiones" (Willkomm \& Lange, 1861-1880, II: 16, sub. Pterocephalus broussonetii Coult.)

\section{EUPHORBIACEAE}

\section{Chamaesyce peplis (L.) Prokh.}

Arraij. PN_Desemb.; Pastizales sobre arenas móviles (playas); raó

\section{Euphorbia boetica Boiss.} (LRA)

Arraij.; Pastizales sobre arenas fijas; oc.; VU

\section{Euphorbia hirsuta L.}

Arraij.; Pastizales graminoides sobre suelos arenosos húmedos; rr.

\section{Euphorbia paralias L.}

Arraij.; Pastizales sobre arenas móviles (playas); rr.

En las prospecciones llevadas a cabo en los últimos años no la hemos vuelto a ver, por lo que creemos que puede haber desaparecido en el área de estudio.

\section{Euphorbia terracina L.}

Arraij. PN_Desemb.; Pastizales sobre arenas fijas; oc.

Mercurialis ambigua L. fil.

PN_Desemb.; Vegetación riparia (tarajales); oc.

\section{FRANKENIACEAE}

Frankenia laevis $L$.

PN_Desemb.; Pastizales halonitrófilos; fr.

Frankenia pulverulenta $\mathrm{L}$.

Arraij. PN_Desemb.; Pastizales halonitrófilos; fr.

\section{GENTIANACEAE}

Centaurium pulchellum (Swartz) Druce

Arraij.; Pastizales sobre suelos arenosos húmedos; ra.

Centaurium tenuiflorum Fritsch

Arraij.; Pastizales sobre suelos húmedos; ra.

Schenkia spicata (L.) G. Mans.

Arraij.; Pastizales sobre suelos arenosos húmedos; rr.

\section{GERANIACEAE}

Erodium aethiopicum (Lam.) Brumh. et Thell. Arraij.; Pastizales sobre arenas fijas; fr.

Erodium chium (L.) Willd.

Pastizales sobre arenas fijas; oc.

Erodium cicutarium (L.) L'Her.

Arraij.; Pastizales sobre arenas fijas; oc.

Erodium laciniatum (Cav.) Willd.

Arraij.; Pastizales sobre arenas fijas; co.

Erodium malacoides (L.) L'Her.

PN_Desemb.; Pastizales sobre arenas fijas; oc.

Erodium moschatum (L.) L'Hér.

Pastizales sobre arenas fijas; oc.

Erodium neuradifolium Delile

Pastizales sobre arenas fijas; oc.

\section{Erodium salzmannii Delile}

Málaga. Málaga. El Arrajainal. Playa de San Julian. 30SUF6958. B. Cabezudo, A. V. Pérez Latorre \& F. Soriguer. MGC 69454

Arraij.; Pastizales sobre arenas fijas; rr.

Erodium propio de arenales costeros muy localizado en Andalucía, citado solo de Cádiz y Málaga (Navarro, 2015). Flora de Andalucía Oriental no recoge su presencia en su territorio. En Málaga, solo lo conocemos de nuestra zona de estudio, sin 
que hayamos encontrado referencias anteriores al pliego citado, exceptuando que, según parece, el material tipo de la especie fue herborizado en estos mismos arenales por Salzmann (Navarro, 2015). En las últimas recolecciones efectuadas no lo hemos vuelto a herborizar.

\section{Geranium dissectum L.}

PN_Desemb.; Vegetación riparia; ra.

\section{GRAMINEAE}

\section{Aegilops geniculata Roth}

Arraij.; Pastizales sobre arenas fijas; oc.

\section{Arundo micrantha Lam.}

Málaga. Málaga. Arroyo Ramírez Bienquerido (encauzamiento). Aguas arriba de Zapata. 30SUF6461. 24/05/2016. Vegetación riparia junto al cauce. F. Soriguer. MGC 82798; Málaga. Zapata. Arroyo Ramírez. Bienqueridos (encauzamiento). 30SUF6461. 17/05/2016. Borde de arroyo. F. Soriguer. MGC 82707

RioG.; Cañaverales en el borde de arroyo; rr.

Caña mucho más escasa que la invasora $A$. donax L. Hasta la fecha parece que esta es la única localidad confirmada en la provincia (en la zona de estudio su presencia ya estaba recogida por Willkomm \& Lange (1861-1880, I: 49) sub. A. plinii Turra), aunque con probabilidad esté presente en más puntos de la provincia.

Avena sterilis L. subsp. sterilis

Arraij.; Pastizales sobre arenas fijas; oc.

\section{Bromus hordeaceus L.}

Arraij. RioG.; Pastizales sobre arenas fijas; oc.

\section{Bromus rigidus Roth}

Arraij.; Pastizales sobre arenas; fr.

Catapodium Ioliaceum (Huds.) Link

Arraij. PN_Desemb.; Pastizales sobre arenas; oc.

Cutandia maritima (L.) Barbey

Arraij.; Pastizales sobre arenas móviles (playas); ra.

\section{Cynodon dactylon (L.) Pers.}

Arraij. PN_Desemb.; Pastizales sobre suelos arenosos húmedos; oc.

\footnotetext{
Dactylis glomerata subsp. hispanica (Roth) Nyman

Arraij.; Pastizales graminoides sobre arenas húmedas; ra.
}

Elymus elongatus (Host) Runemark subsp. elongatus

Málaga. Málaga. Paraje Natural Desembocadura del Guadalhorce. Entre la laguna grande y el brazo derecho del río. 30SUF6959. 21/07/2016. Pastizales perennes en suelos con encharcamiento temporal. F. Soriguer. MGC 84058

PN_Desemb.; Pastizales graminoides sobre suelos húmedos algo salinos; ra.

Gramínea perenne propia de hábitats salobres, rara en Andalucía oriental según Flora de Andalucía Oriental donde solo aparece citada para las comarcas de Guadalquivir y Guadiana menor. Al parecer no citada antes en la provincia de Málaga (Cabezudo et al., 1990).

Elymus farctus (Viv.) Runemark ex Melderis subsp. farctus

Arraij.; Pastizales sobre arenas móviles (playas); co.

Elymus repens (L.) Gould

PN_Desemb.; Pastizales graminoides sobre arenas húmedas; fr.

Festuca arundinacea Schreb.

Arraij. Lags.; Pastizales graminoides sobre arenas húmedas; oc.

Gaudinia fragilis (L.) Beauv.

Lags.; Pastizales sobre suelos húmedos; rr.

Hordeum marinum Hudson

Arraij. PN_Desemb.; Pastizales halófilos; oc.

Hordeum murinum subsp. leporinum (Link) Arcangeli

Arraij.; Pastizales sobre arenas; fr.

Imperata cylindrica (L.) Raeuschel

Arraij. PN_Desemb. RioG.; Pastizales graminoides sobre suelos húmedos algo salinos; oc.

\section{Lolium rigidum Gaudin}

Arraij. PN_Desemb.; Pastizales halófilos y sobre arenas; oc.

\section{Lygeum spartum $\mathrm{L}$.}

Málaga. Málaga. El Arraijanal. Entre la Urbanización Guadalmar y el campo de golf. Entre la playa y los arenales consolidados más interiores. 30SUF6857. 14/04/2016. Mosaico de juncales de Juncus acutus y pastizales halonitrófilos. F. Soriguer. MGC 82275

Arraij. RioG.; Pastizales sobre arenas; rr. 
Gramínea muy característica, abundante en buena parte de Andalucía oriental, pero no en Málaga, en la que solo nos consta una antigua cita de Boissier (1839-1842, II:632) que cita: "las cercanías de Churriana, en zonas secas". Localidad limítrofe con nuestra zona de estudio. Aunque en un principio dudamos del carácter natural de esta población, la cita de Boissier y el hábitat que es propio para el albardín, hacen que lo incluyamos en este catálogo, aunque con reservas.

Melica magnolii Gren. et Godr.

PN_Desemb.; Pastizales sobre suelos arenosos húmedos; rr.

\section{Panicum repens $\mathrm{L}$.}

Arraij.; Pastizales sobre suelos arenosos húmedos; fr.

Parapholis incurva (L.) C. E. Hubb. fr.

Arraij. PN_Desemb.; Pastizales sobre arenas;

\section{Phalaris minor Retz.}

PN_Desemb.; Pastizales sobre arenas; ra.

\section{Phalaris paradoxa $\mathrm{L}$.}

Arraij. PN_Desemb.; Pastizales sobre suelos húmedos; ra.

Phragmites australis (Cav.) Trin. subsp. australis Lags.; Carrizales sobre suelos encharcados o secos; co.

\section{Polypogon maritimus Willd. subsp. maritimus}

Arraij. PN_Desemb.; Pastizales halófilos y sobre arenas; oc.

Polypogon monspeliensis (L.) Desf.

Arraij. PN_Desemb. Lags. RioG.; Pastizales sobre suelos húmedos; oc.

\section{Rostraria cristata (L.) Tzvelev}

PN_Desemb.; Pastizales sobre arenas; fr.

Schismus barbatus (L.) Thell.

Arraij.; Pastizales halonitrófilos sobre suelos arenosos húmedos; ra.

Sorghum halepense (L.) Pers.

PN_Desemb. Lags.; Pastizales graminoides sobre suelos húmedos algo salinos; rr.

Sporobolus pungens (Schreb.) Kunth

Arraij. PN_Desemb.; Pastizales sobre arenas moviles (playas); oc.
Stipa capensis var. pubescens (Ball) Breist. Arraij.; Pastizales sobre arenas; fr.

Trachynia distachya (L.) Link fr.

Arraij. PN_Desemb.; Pastizales sobre arenas;

\section{Triplachne nitens (Guss.) Link}

Málaga. Málaga. El Arraijanal. Cerca del límite con el Campo de Golf. 30SUF6857. 20/04/2016. Suelos con encharcamiento temporal, algo salobres. Arenas-limos. F. Soriguer. MGC 82365; Málaga. Playa del Campo de Golf. 30SUF6857. 28/04/2016. Dunas embrionarias. F. Soriguer. MGC 82499; Málaga. La Cizaña. Entre la Urbanización La Cizaña y la playa. 30SUF6856. 04/05/2016. Arenas litorales. Pastizales sobre arenas. F. Soriguer. MGC 82667; Ibidem. 30SUF6856. 20/05/2016. Juncales y masiegales. Suelos arenosos encharcados. F. Soriguer. MGC 82745

Arraij.; Pastizales sobre arenas; oc.; EN (LRA)

Gramínea anual propia de arenales costeros. No citada para Axarquía en Flora de Andalucía Oriental

Trisetaria panicea (Lam.) Paunero

Arraij.; Pastizales sobre arenas; oc.

Vulpia alopecuros (Schousboe) Dumort. Arraij.; Pastizales sobre arenas; co.

\section{IRIDACEAE}

Gynandriris sisyrinchium (L.) Parl.

Arraij.; Pastizales sobre arenas; oc.

Limniris pseudacorus (L.) Fuss

Arraij. Lags.; Comunidades de grandes helófitos; ra.

\section{JUNCACEAE}

Juncus acutus L. subsp. acutus

Arraij. PN_Desemb. Lags.; Juncales sobre suelos salinos más o menos húmedos; co.

\section{Juncus articulatus $\mathrm{L}$.}

RioG.; Comunidades de grandes helófitos; ra.

Juncus bufonius $\mathrm{L}$.

Arraij. RioG.; Comunidades de helófitos anuales; ra.

Juncus hybridus Brot.

PN_Desemb.; Pastizales sobre suelos arenosos húmedos; rr. 
Junco anual, del complejo grupo de J. bufonius, que no es recogido por Salazar \& Lendinez (2011) en Flora de Andalucía Oriental. Según Romero Zarco (2010) en Flora iberica, nuestro material sí parece corresponder a este taxón.

Juncus inflexus L. subsp. inflexus

Arraij. RioG.; Juncales sobre todo tipo de suelos; oc.

\section{Juncus maritimus Lam.}

Arraij. PN_Desemb. Lags.; Juncales sobre suelos salinos generalmente muy húmedos; fr.

Juncus subulatus Forssk.

Arraij. PN_Desemb. Lags. RioG.; Comunidades higrófilas de suelos encharcados; co.

\section{LABIATAE}

\section{Lycopus europaeus $\mathrm{L}$.}

Arraij.; comunidades higrófilas; ra.

Mentha rotundifolia (L.) Hudson

RioG.; comunidades higrófilas; oc.

Stachys ocymastrum (L.) Briq.

Arraij. PN_Desemb. RioG.; Pastizales sobre arenas; oc.

\section{LEGUMINOSAE}

\section{Astragalus boeticus $\mathrm{L}$.}

Arraij.; Pastizales sobre arenas; rr.

Dorycnium rectum (L.) Ser. in DC.

PN_Desemb. RioG.; Vegetación riparia en el borde del río; ra.

\section{Glycyrrhiza glabra L.}

PN. Desemb.; Pastizales en bordes de antiguas acequias; ra.

\section{Hippocrepis multisiliquosa $\mathrm{L}$.}

Arraij.; Pastizales sobre arenas; oc.

\section{Lotus arenarius Brot.}

Arraij.; Pastizales sobre arenas; fr.

Lotus castellanus Boiss. et Reut. PN_Desemb.; Pastizales halófilos; rr.

\section{Lotus ornithopodioides L.}

PN_Desemb.; Pastizales sobre arenas; rr.

\section{Lupinus cosentinii Guss}

Arraij.; Pastizales sobre arenas; oc.

Altramuz muy raro en Andalucía oriental, donde solo está presente en la provincia de Málaga, (De las Heras \& Muñoz Rodriguez, 2011; Castroviejo \& Pascual, 1999) y por lo que sabemos solo en los arenales de nuestra área de estudio. Puede que, debido a la abundancia de este altramuz en estos arenales, se deba el topónimo "Los Chochales" a los terrenos del actual campo de golf (mapa 1:25.000. IGN), ya que, en Málaga, a los altramuzes se le denomina "Chochos".

Medicago littoralis Rohde ex Loisel.

Arraii.; Pastizales sobre arenas; co.

Medicago marina L.

Arraij.; Pastizales sobre arenas moviles (playas); co.

Ononis ramosissima Desf.

Arraij. PN_Desemb.; Pastizales sobre arenas fijas; co.

\section{Ononis variegata $\mathrm{L}$.}

Arraij.; Pastizales sobre arenas móviles (playas); ra.

\section{Trifolium tomentosum $\mathrm{L}$.}

Arraij.; Pastizales sobre arenas; oc.

Vicia cordata Hoppe

PN_Desemb.; Pastizales sobre arenas; rr.

\section{LEMNACEAE}

\section{Lemna gibba L.}

PN_Desemb. Lags.; Vegetación hidrofítica flotante de agua dulce; oc.

\section{LILIACEAE}

Allium ampeloprasum $\mathrm{L}$.

Arraij. RioG.; Pastizales sobre arenas; oc.

Allium roseum $\mathrm{L}$.

Arraij. RioG.; Pastizales sobre arenas; oc.

Asphodelus tenuifolius Cav.

Arraij.; Pastizales sobre arenas fijas; co.

Dipcadi serotinum (L.) Medicus subsp. serotinum Arraij.; Pastizales sobre arenas; oc.

Muscari comosum (L.) Mill.

Arraij.; Pastizales sobre arenas; ra. 
Muscari parviflorum Desf.

Arraij.; Pastizales sobre arenas; rr.

Citada recientemente en la zona (CasimiroSoriguer Solanas et al., 2013). Es de las poquísimas poblaciones que se conocen de esta planta en la Península Ibérica, donde unicamente se conoce de la provincia de Málaga (Suarez-Santiago \& Blanca, 2013).

Urginea maritima (L.) Baker

Arraij.; Pastizales sobre arenas; oc.

\section{LINACEAE}

\section{Linum maritimum $\mathrm{L}$.}

Dehesilla de Málaga; Juncales sobre arenas; rr. (¿extinto?)

Málaga. Dehesilla. 8/1934. M. Laza. MA 72968

Lino perenne propio de saladares. En Málaga solo nos consta como localidad fiable nuestra área de estudio donde además del pliego testigo que citamos y hemos podido consultar, tenemos referencias anteriores de Boissier (1939-1842, II:108) de la Dehesilla de Málaga. A pesar de haberlo buscado no hemos vuelto a encontrarlo.

Linum setaceum Brot.

Arraij.; Pastizales sobre arenas; fr.

\section{LYTHRACEAE}

\section{Lythrum hyssopifolia L.}

Arraij.; Pastizales sobre arenas húmedas; rr.

Lythrum junceum Banks et Sol.

Arraij. Lags. RioG.; Comunidades de hidrófitos de aguas dulces; ra.

\section{Lythrum salicaria L.}

PN_Desemb.; Vegetación riparia; ra.

\section{MALVACEAE}

\section{Lavatera cretica L.}

Arraij. PN_Desemb.; Pastizales sobre arenas húmedas; ra.

\section{Malva parviflora $\mathrm{L}$.}

Arraij. PN_Desemb.; pastizales halonitrófilos; ra.

\section{NAJADACEAE}

\section{Najas marina L.}

Málaga. Málaga; Desembocadura del Guadalhorce. 30SUF7059. 4/6/1997. R. Conde
Álvarez \& R. Orozco. MGC 44946

PN_Desemb.; Vegetación hidrofítica sumergida de aguas hiposalinas o dulces; $r$ r.

La única cita malagueña de este macrófito corresponde a la Desembocadura del río Guadalhorce (Nieto Caldera et al., 1997). Nosotros no la hemos vuelto a encontrar pese a prospectar en todas las lagunas del actual Paraje Natural. En Málaga solo la hemos vuelto a ver en las lagunas artificiales del Parque Tecnológico de Andalucía (MGC 81695).

\section{ONAGRACEAE}

Epilobium tetragonum subsp. tournefortii (Michalet) Rouy et E.G. Camus

Málaga. Málaga. Lagunajo del Campamento Benítez. 30SUF6757. 10/10/2017. Comunidades de helófitos y carrizales. F. Soriguer, J. GarcíaSánchez \& M. Pulgar. MGC 87344

Lags.; Comunidades higrófilas; rr.

No citada en Axarquía en Flora de Andalucía Oriental.

\section{OROBANCHACEAE}

Orobanche amethystea Thuill. subsp. amethystea Arraij.; Pastizales sobre arenas; ra. Probablemente sobre Eryngium maritimum $\mathrm{L}$.

Orobanche crenata Forssk.

PN_Desemb.; Pastizales sobre arenas; ra.

Orobanche minor Sm.

Arraij.; Pastizales sobre arenas; ra.

Orobanche mutelii F.W. Schultz

Arraij.; Pastizales sobre arenas; ra.

\section{PAPAVERACEAE}

\section{Fumaria capreolata L.}

Arraij. RioG.; Juncales sobre arenas; ra.

Glaucium flavum Crantz

Arraij. PN_Desemb.; Pastizales sobre arenas; ra.

\section{PLANTAGINACEAE}

Plantago albicans $L$.

Arraij. RioG.; Pastizales sobre arenas fijas; co.

Plantago bellardii All.

Arraij.; Pastizales sobre arenas fijas; ra. 
Plantago coronopus $\mathrm{L}$.

Arraij. PN_Desemb.; Pastizales halonitrófilos y sobre suelos arenosos húmedos; oc.

\section{Plantago major L.}

Río G.; Pastizales nitrificados sobre suelos húmedos; oc.

\section{PLUMBAGINACEAE}

Limonium sinuatum (L.) Mill.

PN_Desemb.; Pastizales halonitrófilos; ra.

\section{POLYGONACEAE}

\section{Emex spinosa (L.) Campd.}

Arraij.; Pastizales ruderalizados sobre arenas; oc.

\section{Polygonum lapathifolium $\mathrm{L}$.}

PN_Desemb.; Vegetación riparia; co.

\section{Polygonum maritimum L.} oc.

Arraij. PN_Desemb.; Pastizales sobre arenas;

Polygonum salicifolium Brouss. ex Willd. Lags. RioG.; Comunidades higrófilas; oc.

Rumex bucephalophorus subsp. gallicus (Steinh.) Rech. Fil.

Arraij. RioG.; Pastizales sobre arenas; oc.

\section{Rumex conglomeratus Murray}

Arraij. PN_Desemb. RioG.; Comunidades higrófilas; oc.

\section{Rumex crispus L.}

PN_Desemb.; Comunidades higrófilas; oc.

\section{Rumex palustris $\mathrm{Sm}$.}

Málaga. Málaga. El Arraijanal. Arroyo en el límite oriental del Campo de Golf. 30 SUF6858. 21/07/2016. Arroyo. Vegetación higrófila. F. Soriguer. MGC 84072; Ibidem. 30SUF6856. 04/05/2016. Vegetación higrófila. F. Soriguer. MGC 82681

Arraij.; Comunidades higrófilas; rr.; DD (LRA)

Al parecer no citado con anterioridad en la provincia de Málaga (López Gonzalez, 1990; Diez Garretas, 1977; Salinas, 2011).

\section{POTAMOGETONACEAE}

\section{Potamogeton pectinatus $\mathrm{L}$.}

Málaga. Málaga; Desembocadura del
Guadalhorce. 30SUF7059. 4/6/1997. R. Conde Álvarez \& R. Orozco. MGC 44935; Ibidem. Laguna Escondida. 30SUF6959. 19/5/2015. F. Soriguer. MGC 80892

PN_Desemb.; Vegetación hidrofítica sumergida de aguas hiposalinas o dulces; rr.

Hidrófito muy localizado en la zona de estudio. Citado anteriormente por Nieto Caldera et al., (1997). Hemos observado densas poblaciones en los canales artificiales del cercano Parque Tecnológico de Andalucía (MGC 81697).

\section{Potamogeton sp. (sect. Potamogeton)}

Málaga. Málaga. Río Guadalhorce. Vado del Tarajal (puente del Rey). 30SUF6462. 10/10/2017. Comunidades de hidrófitos sumergidos y flotantes. Borde de río. Agua corriente. F. Soriguer, J. GarcíaSánchez \& M. Pulgar. MGC 87350

Rio G.; Vegetación hidrofítica sumergida; rr.

Seguramente el material recolectado corresponda a $P$. nodosus Poir., pero al no presentar estructuras reproductivas no se ha podido identificar a nivel específico.

\section{PRIMULACEAE}

\section{Anagallis foemina Mill.}

Arraij.; Pastizales sobre arenas; co.

\section{Samolus valerandi $\mathrm{L}$.}

Lags.; Comunidades higrófilas; oc.

\section{RANUNCULACEAE}

\section{Delphinium nanum DC.}

Arraij.; Pastizales sobre arenas; fr.

\section{Ranunculus trichophyllus Chaix subsp. trichophyllus}

Málaga. Málaga. Lagunajo entre el Campamento Benítez y el campo de golf. 30SUF6757. 03/05/2017. Comunidades de hidrófitos sumergidos. F. Soriguer \& J. GarcíaSánchez. MGC 85250

Lags.; Vegetación hidrofítica flotante de agua dulce; rr.

Ranunculus trilobus Desf.

Lags.; Pastizales sobre suelos húmedos; oc.

\section{ROSACEAE}

\section{Rubus ulmifolius Schott}

PN. Desemb., Lags., Rio G.; Vegetación riparia; co.

Observada, pero sin pliego de herbario 


\section{RUBIACEAE}

\section{Crucianella maritima L.}

Málaga. Málaga. El Arraijanal. Arroyo en el límite Este del campo de golf. 30SUF6857. 14/04/2016. Arenales consolidados. F. Soriguer. MGC 82277

Arraij.; Pastizales sobre arenas; rr.

Galium aparine L. subsp. aparine

PN_Desemb. Lags.; Comunidades de helófitos (bayuncales) en aguas dulces; oc.

Galium palustre var elongatum (C. Presl) Rchb. fil.

Málaga. Málaga. Entre Campo de Golf y Campamento Benítez. 30SUF65. 9/6/1980. . J. M. Nieto. MGC 8060; Málaga. Lagunajo entre el Campamento Benítez y el campo de golf. 30SUF6757. 03/05/2017. Formaciones de helófitos en el interior de la laguna. F. Soriguer \& J. GarcíaSánchez. MGC 85245

Lags.; Comunidades de helófitos (bayuncales) en aguas dulces; rr.

\section{RUPPIACEAE}

\section{Ruppia maritima L.}

Málaga. Málaga. Desembocadura del río Guadalhorce. Desembocadura del arroyo junto al brazo derecho del río. 30SUF6858. 21/07/2016. Vegetación halofítica hidrofítica sumergida. F. Soriguer. MGC 84075; ibidem. 30 SUF7059. 4/6/1997. R. Conde Álvarez \& R. Orozco. MGC 44947; Ibididem. Entre la 'Laguna Grande' y el 'Brazo Viejo'. 30SUF7059. 19/5/2015. F. Soriguer. MGC 80893; Ibidem. Laguna Escondida. 30SUF6959. 19/5/2015. F. Soriguer. MGC 80891; Ibidem. Pequeña laguna entre la Laguna Grande y la playa. 30SUF6959. 27/4/2015. F. Soriguer \& J. M. Nieto. MGC 80748

PN_Desemb.; Vegetación hidrofítica sumergida de aguas salinas; ra.; DD (LRA)

\section{SALICACEAE}

\section{Populus alba L.}

PN_Desemb. RioG.; Vegetación riparia; fr.

\section{Salix purpurea L.}

PN_Desemb. RioG.; Vegetación riparia; fr.

\section{SCROPHULARIACEAE}

Linaria pedunculata (L.) Chaz.

Arraij. PN_Desemb. Lags.; Pastizales sobre arenas moviles (playas); co.; VU (LRA)

Misopates orontium (L.) Raf.

Arraij.; Pastizales sobre arenas; oc.

TAMARICACEAE

Tamarix africana Poir.

Arraij. PN_Desemb.; Vegetación riparia (tarajales); co.

Tamarix canariensis Willd.

Arraij. RioG.; Vegetación riparia (tarajales); oc.

Tamarix gallica $\mathrm{L}$.

PN_Desemb. RioG.; Vegetación riparia (tarajales); oc.

\section{TYPHACEAE}

Typha angustifolia L.

PN_Desemb.; Formaciones de grandes heliofilos en aguas dulces; fr.

\section{UMBELLIFERAE}

Apium nodiflorum (L.) Lag.

Arraij. PN_Desemb. RioG.; Formaciones de heliofilos en aguas dulces; ra.

Cachrys sicula L.

Arraij.; Pastizales sobre arenas; ra.

\section{Eryngium campestre $\mathrm{L}$.}

Arraij.; Pastizales sobre arenas fijas; rr.

\section{Eryngium maritimum $\mathrm{L}$.}

Arraij.; Pastizales sobre arenas moviles (playas); ra.

Oenanthe globulosa L.

Lags.; Comunidades higrófilas; rr.

Pseudorlaya pumila (L.) Grande

Arraij. PN_Desemb.; Pastizales sobre arenas; fr.

\section{VERBENACEAE}

Vitex agnus-castus $\mathrm{L}$.

Arraij. PN_Desemb. RioG.; Vegetación riparia; rr

\section{ZANNICHELLIACEAE}

Zannichellia sp.

Málaga. Málaga. Río Guadalhorce. Vado del 
Tarajal (puente del Rey). 30SUF6462. 10/10/2017. Comunidades de hidrófitos sumergidos y flotantes. Borde de río. Agua corriente. F. Soriguer, J. GarcíaSánchez \& M. Pulgar. MGC 87351

Rio G.; comunidades de hidrófitos sumergidos; rr.

El material herborizado no ha podido ser identificado a nivel específico al no poseer estructuras reproductivas. De nuestra zona de estudio solo tenemos referencias de este género de Z. contorta(Desf.) Cham. \& Schltdl. como Z. macrostemon Gay. en Willkomm \& Lange (18611880, I: 26)

\section{ZOSTERACEAE}

\section{Zostera marina L.}

Málaga. Málaga. La Misericordia. 30SUF7036060550. 5/5/1976. A. M. Hernández. MGC 3313

PN_Desemb.; Vegetación de fondos marinos; rr.; VU (LRA), (LAESRPE)

El material del único pliego testigo de la zona parece haber sido recolectado arribado en la playa por lo que no sabemos en qué estado pueda estar la población de esta fanerógama marina en la zona.

\section{ZYGOPHYLLACEAE}

\section{Tribulus terrestris $\mathrm{L}$.}

Arraij.; Pastizales sobre arenas; oc.

\section{Catálogo flora aloctona}

Aunque sin pliego testigo también hemos observado en la zona de forma más o menos puntual:

Washintonia spp., Phoenix spp., Acacia spp., Parkinsonia spp. y Carpobrotus spp.

\section{AIZOACEAE}

Galenia pubescens (Eckl. et Zeyh.) Druce

PN. Desemb., Arraij.; Comunidades ruderales, pastizales halonitrófilos y sobre arenas; co.; metáfito

Citada en la provincia de Málaga en un primer momento como G. secunda (L.) Fil. Sonder (Casimiro-Soriguer Solanas \& Pérez Latorre, 2008), se corrige ahora todo el material de la zona a G. pubescens, siguiendo a Veerlove \& Sánchez Gullón (2008). Es en nuestra opinión la especie alóctona invasora más peligrosas en la zona desde el punto de vista medio ambiental. Forma auténticos tapices rastreros monoespecíficos de decenas de metros cuadrados en todo tipo de suelos, también la hemos visto como trepadora en tarajales. Es en el Paraje Natural donde es más abundante y aunque es allí donde su crecimiento es más feraz, es su presencia en lugares ruderalizados cada vez mayor. En comunidades psammícolas e higrófilas la hacen sumamente dañina para estos hábitats, ya de por sí muy amenazados por la actividad humana. De hecho, la peligrosidad de esta especie y la necesidad de su control ya fue puesta de manifiesto por García de Lomas et al., (2010).

Aunque esta planta está dentro de las especies de flora exótica invasora sobre las que se han realizado trabajos de eliminación en Andalucía por parte de la Junta de Andalucía (Web de la Consejería de Medio Ambiente y Ordenación del Territorio), no tenemos constancia de que se haya realizado ninguna actuación de control (aunque si alguna actuación puntual de experimentación metodológica) en el área estudiada, ni en el área protegida del Paraje Natural ni en la Laguna de los Prados (incluida en IHA) donde también está instalada.

\section{AMARANTHACEAE}

\section{Amaranthus albus $\mathrm{L}$.}

Arraij. PN_Desemb.; Pastizales ruderales sobre arenas; oc.; metáfito epecófito

\section{Amaranthus viridis $\mathrm{L}$.}

PN_Desemb.; Pastizales ruderales sobre arenas; oc.; metáfito epecófito

\section{ASCLEPIADACEAE}

\section{Asclepias fruticosa $\mathrm{L}$.}

Arraij.; Pastizales ruderales sobre arenas; metáfito epecófito

\section{BORAGINACEAE}

\section{Heliotropium curassavicum $\mathrm{L}$.}

Arraij. PN_Desemb.; Pastizales halonitrófilos; oc.; metáfito

Citada por primera vez en la zona por Diez Garretas (1977) donde está plenamente asentada, no parece afectar negativamente a las especies autóctonas, aunque pueda competir con las especies halófilas y halonitrófilas por los cada vez más reducidos enclaves conservados.

\section{CAPRIFOLIACEAE}

Lonicera japonica Thunb.

Arraij.; Vegetación riparia; ra.; metáfito agriófito 


\section{COMPOSITAE}

Arctotheca calendula (L.) Levyns

Arraij. PN_Desemb.; Pastizales sobre arenas móviles (playas); oc.; metáfito agriófito

\author{
Symphyotrichum squamatun (Sprengel) G.L. \\ Nesson \\ metáfito \\ PN_Desemb.; comunidades higrófilas; oc.;
}

\section{Cotula coronopifolia L.}

Málaga. Málaga. Paraje Natural Desembocadura del Guadalhorce. Brazo oriental del encauzamiento. 30SUF7059. 3/6/2015. F. Soriguer. MGC 81211; Málaga. Paraje Natural Desembocadura del río Guadalhorce. 30SUF6962059720. F. Casimiro Soriguer Solanas. MGC 54109

PN_Desemb.; Vegetación halófila acuática; ra.; metáfito agriófito

De la presencia de esta compuesta anual en la provincia de Málaga solo teníamos constancia de una recolección anterior a este trabajo (MGC 54109), pliego en el que se basa la cita para Axarquía de Flora de Andalucía Oriental. Ahora la hemos vuelto a ver de nuevo en la Desembocadura del río Guadalhorce, donde aparece de forma permanente, aunque su presencia depende del nivel del agua, por lo que su población es muy fluctuante. De momento no parece que suponga un peligro para la flora autóctona. Sería una especie nueva a incluir en el catálogo de metafitos de la provincia de Málaga (Casimiro-Soriguer Solanas \& Perez Latorre, 2008).

\section{Eclipta prostrata (L.) L.}

Málaga. Málaga. El Arraijanal. Arroyo en el límite oriental del campo de golf. 30 SUF6857. 10/10/2017. Comunidades de anuales en suelos húmedos sometidos a la influencia marina. $F$. Soriguer, J. García-Sánchez \& M. Pulgar. MGC 87346; Málaga. Río Guadalhorce. Vado del Tarajal (puente del Rey). 30SUF6462. 10/10/2017. Comunidades de helófitos en el borde del cauce. F. Soriguer, J. García-Sánchez \& M. Pulgar. MGC 87356

Río G.; Arrii.; Comunidades higrófilas en suelos encharcados; fr.; metáfito agriófito

Citada en una sola localidad en Málaga, en el río Guadalmedina, en los pantanos del Agujero y Limonero (Pérez Latorre et al., 2008). En el área de estudio se presenta abundante en los cauces de arroyos y del propio río Guadalhorce cuando el estiaje es más acusado. No sabemos cómo puede afectar a las comunidades de anuales asociadas a estos hábitats, pero llega a formar céspedes muy densos

\section{CRUCIFERAE}

Coronopus didymus (L.) Sm.

Arraij. PN_Desemb.; Pastizales sobre arenas; ra.; metáfito epecófito

\section{CYPERACEAE}

Cyperus involucratus Rottb.

Málaga. Málaga. San Julián. Entre el vivero del icona y el polígono industrial. Lagunajo del vivero. 30SUF6758. 29/03/2017. Formaciones de helófitos y grandes carices sobre suelos encharcados arcillosos. F. Soriguer \& J. García-Sánchez. MGC 84812

Lags.; Comunidades de grandes cárices asociados a aguas dulces; ra.; metáfito agriófito

El papiro solo lo habíamos observado en Málaga de forma puntual y esporádica, en las cercanías de la capital, no estando incluido en el catálogo de metáfitos de la provincia de Málaga (Casimiro-Soriguer Solanas \& Pérez Latorre, 2008). La población asociada a la laguna del Vivero de San Julián tiene una densidad y porte considerable, compitiendo directamente con la población de Carex riparia.

\section{GRAMINEAE}

\section{Arundo donax $\mathrm{L}$.}

Arraij. PN_Desemb. Lags., Río G.; Comunidades riparias; co.; metáfito agriofito

Cortaderia selloana (Schult. et Schult. f.) Asch. et Graebn.

PN_Desemb.; herbazales nitrófilos; ra.; diáfito

Echinochloa crus-galli (L.) P.Beauv.

Arraij.; Comunidades de helófitos; rr.; diáfito

\section{Eragrostis barrelieri Daveau}

Málaga. Málaga. La Cizaña. Entre la Casa de la Cizaña y la Urbanización La Cizaña. 30SUF6756. 30/03/2016. Arenales litorales consolidados. Pastizales. F. Soriguer. MGC 81921; Ibidem. 30SUF6856. 06/04/2016. Arenales consolidados. Pastizales. J. García-Sánchez \& F. Soriguer. MGC 82097

Arraij.; Juncales y herbazales halófilos sobre suelos arenosos humedos; ra; metáfito agriófito

No citada en Flora de Andalucía Oriental para Axarquía. Novedad para el catálogo de metáfitos de la provincia de Málaga. 


\section{Paspalum vaginatum $\mathrm{Sw}$.}

Arraij. PN_Desemb. Lags.; Río G.; Pastizales sobre suelos encharcados; fr.; metáfito agriófito

Muy común en todas las zonas húmedas de nuestra zona de estudio formando céspedes.

\section{Stenotaphrum secundatum (Walter) Kuntze}

Málaga. Málaga. El Arraijanal. 30SUF6858. 10/10/2017. Arenales litorales. Arenas consolidadas. F. Soriguer, J. García-Sánchez \& M. Pulgar. MGC 87339

Arraij.; Pastizales sobre suelos arenosos húmedos; oc.; metáfito agriófito

Citada en Flora de Andalucía Oriental solo para Alpujarras. En nuestra zona de estudio tiene un comportamiento invasor claro, formando densos céspedes casi monoespecíficos. Novedad para el catálogo de metáfitos de la provincia de Málaga.

\section{MYOPORACEAE}

Myoporum laetum G. Forst.

PN_Desemb.; zonas ruderales; ra.; metáfito epecófito

\section{MYRTACEAE}

\section{Eucalyptus camaldulensis Dehnh}

Arraij., PN_Desemb., Lags., Río Comunidades riparias; co.; metáfito agriófito

\section{ZYGOPHYLLACEAE}

\section{Zygophyllum fabago $\mathrm{L}$.}

PN_Desemb.; comunidades ruderales; ra.; metáfito epecófito

\section{Agradecimientos}

Agradecemos a Baltasar Cabezudo como director científico del Herbario MGC y como editor de la revista su disposición para el desarrollo y evaluación de este trabajo. Al Herbario MGC de los Servicios Centrales de Apoyo a la Investigación (SCAI) de la Universidad de Málaga por facilitar todo el material solicitado y al Herbario MA del Real Jardín Botánico de Madrid por proporcionar una imagen del pliego MA 72968 de Linum maritimum L. A la Consejería de Medio Ambiente de la Junta de Andalucía por proporcionar los permisos necesarios para trabajar en el Paraje Natural y a Antonio Tamayo, Agente de Medio Ambiente de la Junta de Andalucía por su ayuda en la localización de algunas de las zonas de estudio.

\section{Referencias}

Asensi, A. \& Nieto Caldera, J.M. (1981). Vegetación acuática, halófila y halonitrófila de la provincia de Málaga. Trabajos y Monografías del Departamento de Botánica de Málaga, 2, 105-122.

Blanca, G. (2011). Hedypnois Mill. En Blanca, G., Cabezudo, B., Cueto, M., Morales Torres \& Salazar, C. (Eds.). Claves de la Flora Vascular de Andalucía Oriental (pp. 590-591). Granada, España. Universidades de Granada, Almería, Jaén y Málaga.

Blanca, G., Cabezudo, B., Cueto, M., Morales Torres \& Salazar, C. (Eds.). (2011). Claves de la Flora Vascular de Andalucía Oriental. Granada, España. Universidades de Granada, Almería, Jaén y Málaga.

Boissier, E. (1839-1842). Voyage Botanique dans le Midi de l'Espagne Pendant l'année 1837. París, Francia.

Cabello, J. (2011). Sarcocornia A.J. Scott En Blanca, G., Cabezudo, B., Cueto, M., Morales Torres \& Salazar, C. (Eds.). Claves de la Flora Vascular de Andalucía Oriental (pp. 226-227). Granada, España. Universidades de Granada, Almería, Jaén y Málaga.

Cabezudo, B., Devesa, J.A., Tormo, R., Vázquez, F. \& Nieto-Caldera, J.M. (1990). Catálogo de las Gramíneas Malacitanas. Acta Bot. Malacitana, 15, 91123.

Cabezudo, B., Arenas Posada, J.A., García Martín, F. \& Nieto Caldera, J.M. (1992). Catálogo de las umbelíferas (Apiaceae) malacitanas (Málaga, España). Acta Bot. Malacitana, 17, 145-166.

Cabezudo, B., Talavera, S., Blanca, G., Salazar, C., Cueto, M., Valdés, A., Hernández, J.E., Herrera, C.M., Rodríguez, C. \& Navas, D. (2005). Lista roja de la Flora Vascular de Andalucía. Sevilla, España. Consejería Medio Ambiente. Junta de Andalucía.

Casimiro-Soriguer Solanas, F. (2009). Nuevos datos sobre Lonicera biflora Desf., en la provincia de Málaga (España). Lagascalia, 29, 279-281.

Casimiro-Soriguer Solanas, F. \& Pérez Latorre, A.V. (2008). Aproximación al conocimiento de la flora alóctona de la provincia de Málaga (España): catálogo de metáfitos. Acta bot. Malacitana, 33, 373-382.

Casimiro-Soriguer Solanas, F., Pérez Latorre, A.V., Hidalgo Triana, N., García Sanchez, J., Pavón, M. \& Cabezudo, B. (2013). Algunas plantas interesantes de Andalucía oriental II. (Málaga-Granada). Acta Bot. Malacitana, 38, 202-207.

Castroviejo, S. (1990). Sarcocornia A.J. Scott En Castroviejo, S. et al. (Eds.). Flora iberica 2 (pp. 528531). Madrid, España. Real Jardín Botánico. CSIC.

Castroviejo, S. (coord. Gen.) (1986-2017). Flora iberica 1-16(2), 17-18, 20-21. Madrid, España. Real Jardín Botánico. CSIC.

Castroviejo, S. \& Pascual, H. (1999). Lupinus L. En Castroviejo, S. et al. (Eds.). Flora iberica 7(1) (pp. 252260). Madrid, España. Real Jardín Botánico. CSIC.

Castroviejo, S. \& Coello, P. (1980). Datos cariológicos y taxonómicos sobre las Salicorniinae A.J. Scott Ibéricas. Anales Jard. Bot. Madrid, 37(1), 41-73.

De las Heras, M.A. \& Muñoz Rodríguez, A.F. (2011). Lupinus L. En G. Blanca, G., Cabezudo, B., Cueto M., Morales Torres, C. \& Salazar, C. (Eds.). Claves de la Flora vascular de Andalucía Oriental (pp. 284285). Granada, España. Universidades de Granada, Almería, Jaén y Málaga. 
Dana, E., Sanz-Elorza, M., Vivas, S. \& Sobrino, E. (2005). Especies vegetales invasoras en Andalucía. Sevilla, España. Consejería de Medio Ambiente. Junta de Andalucía.

Devesa, J.A. \& Viera, M.C. (2001). Viajes de un botánico sajón por la Península Ibérica (H.M. Willkomm, 18211895). Cáceres, España. Universidad de Extremadura. Servicio de publicaciones.

Díez Garretas, B. (1977). Flora y vegetación del litoral marino de las provincias de Málaga y Granada (Tesis doctoral). Universidad de Granada, Granada.

Hardion, L., Verlaque, R., Callmander, M.W. \& Vila, B. (2012). Arundo micrantha Lam. (Poaceae), the correct name for Arundo mauritanica Desf. and Arundo mediterranea Danin. Candollea, 67, 131-135.

GBIF (Global Biodiversity Information Facility). Portal español de datos de GBIF, http://datos.gbif.net, 24/01/2013. Herbarios y bases de datos consultadas: MA, GDA, GDAC, COA, COFC, JBAG, SALA, VAL, BC y Anthos.

Laza-Palacios, M. (1944). Estudios sobre la flora andaluza. Anal. Real Acad. Farm., 2, 156-556.

Luceño, M. \& M. Escudero (2007). Carex L., Sect. Tumidae. En Castroviejo, S. et al. (Eds.). Flora iberica 18 (pp. 110-250). Madrid, España. Real Jardín Botánico. CSIC.

Navarro, C. (2015). Erodium L`Her. En Castroviejo, S. et al. (Eds.). Flora iberica 9 (pp. 316-372). Madrid, España. Real Jardín Botánico. CSIC

Nieto Caldera J.M., Conde, R.M., Arrebola Bautista, M.A. \& Flores Moya, A. (1997). Nuevas citas de macrófitos acuáticos de las zonas húmedas de la provincia de Málaga. Acta Bot. Malacitana, 22, 247-248.

Pedrol, J. \& Castroviejo, S. (1990). Suaeda Scop. En Castroviejo, S. et al. (Eds.). Flora iberica 2 (pp. 536541). Madrid, España. Real Jardín Botánico. CSIC.

Pérez Latorre, A. V. \& Cabezudo, B. (2002). La flora y el paisaje vegetal de la provincia de Málaga: importancia y conservación. Jábega, 90, 25-39.

Rivas-Martínez S., Penas, A., Díaz Gonzalez, T.E., Cantó, P., del Río, S., Costa, J. C., Herrero, L. \& Molero, J. (2017). Biogeographic Units of the Iberian Peninsula and Balearic Islands to District Level. En Loidi, J. (Ed.). The Vegetation of the Iberian Peninsula (Vol. I) (pp. 131-188). Cham, Swistzerland. Ed. Springer.

Rivas Goday, S. \& Rivas Martínez, S. (1959). Acerca de la Ammophiletea del Este y Sur de España. Anales Inst. Bot. Cavanilles, 16, 549-564

Romero, A.T. (2011). Cutandia Willk. En G. Blanca, G., Cabezudo, B., Cueto M., Morales Torres, C. \& Salazar, C. (Eds.). Claves de la Flora vascular de Andalucía Oriental (p. 126). Granada, España. Universidades de Granada, Almería, Jaén y Málaga.

Romero Zarco, C. (2010). Juncus L. En Castroviejo, S. et al. (Eds.). Flora iberica 17 (pp. 124-187). Madrid, España. Real Jardín Botánico. CSIC

Romero Zarco, C. (2015). Las gramíneas de la Península
Ibérica e Islas Baleares. Colección Monografias de Botánica Ibérica, 15. Jaca, España. Jolube Consultor Botanico y Editor.

Salazar, C. (2011). Cladium (L.) Pohl En G. Blanca, G., Cabezudo, B., Cueto M., Morales Torres, C. \& Salazar, C. (Eds.). Claves de la Flora vascular de Andalucía Oriental (p. 103). Granada, España. Universidades de Granada, Almería, Jaén y Málaga.

Salazar, C. \& J. Quesada (2011). Carex L. En G. Blanca, G., Cabezudo, B., Cueto M., Morales Torres, C. \& Salazar, C. (Eds.). Claves de la Flora vascular de Andalucía Oriental (pp. 103-108). Granada, España. Universidades de Granada, Almería, Jaén y Málaga

Salazar, C. (2011). Eleocharis R.Br. En G. Blanca, G., Cabezudo, B., Cueto M., Morales Torres, C. \& Salazar, C. (Eds.). Claves de la Flora vascular de Andalucía Oriental (pp. 102-103). Granada, España. Universidades de Granada, Almería, Jaén y Málaga.

Salazar, C. \& L. Lendínez (2011). Juncus L. En G. Blanca, G., Cabezudo, B., Cueto M., Morales Torres, C. \& Salazar, C. (Eds.). Claves de la Flora vascular de Andalucía Oriental (pp. 96-98). Granada, España. Universidades de Granada, Almería, Jaén y Málaga.

Silvestre, S. (1987) Carex. En Valdés, B., Talavera, S. \& Galiano, E.F. (Eds.). Flora Vascular de Andalucía Occidental (Vol. III). Barcelona, España. Ed. Ketres.

Silvestre, S. (2012) Calystegia. En Castroviejo, S. et al. (Eds.). Flora iberica 11 (pp. 273-278). Madrid, España. Real Jardín Botánico. CSIC.

Suarez Santiago V.N. \& Blanca, G. (2013). Muscari. En Castroviejo, S. et al. (Eds.). Flora iberica 20 (pp. 172184). Madrid, España. Real Jardín Botánico. CSIC

Talavera, S. \& Muñoz Garmendia, F. (1988). Sinopsis del género Silene L. (Caryophyllaceae) en la Península Ibérica y Baleares. Anales Jard. Bot. Madrid, 45(2), 407-460.

Talavera, S., Talavera, M. \& Sánchez, C. (2015). Los géneros Thrincia Roth y Leontodon L. (Compositae) en Flora Iberica. Acta Bot. Malacitana, 40, 344-364.

Talavera, M., Berjano, R., Jiménez, F.J. \& Talavera, S. (2016). Consideraciones sobre el género Hedypnois (Asteraceae) y tipificación de dos especies descritas por Willdenow. Acta Bot. Malacitana, 41, 329-334.

Valdés, B. (2012) Anchusa L. En Castroviejo, S. et al. (Eds.). Flora iberica 11 (pp. 349-363). Madrid, España. Real Jardín Botánico. CSIC.

Valdés, B., Talavera, S. \& Galiano, E.F. (Eds.) (1987). Flora Vascular de Andalucía Occidental. Barcelona, España. Ed. Ketres.

Verloove, F. \& Sánchez Gullón E. (2008). New records of interesting xenophytes in the Iberian Peninsula. Acta Bot. Malacitana, 33, 147-167.

Willkomm, M. \& Lange, J. (1861-1880). Prodromus Florae Hispanicae. Stuttgart, Alemania.

Willkomm, M. (1893). Supplementum Prodromi Florae Hispanicae. Stuttgart, Alemania. 